\title{
Efficient generation of entangled photons by cavity QED
}

\author{
X.L. Zhang ${ }^{1,2}$, M. Feng ${ }^{1}$ * and K.L. Gao ${ }^{1}$ \\ ${ }^{1}$ State Key Laboratory of Magnetic Resonance and Atomic and Molecular Physics, \\ Wuhan Institute of Physics and Mathematics, \\ Chinese Academy of Sciences, Wuhan 430071, China and \\ ${ }^{2}$ Graduate School of the Chinese Academy of Science, Bejing 100049, China
}

\begin{abstract}
A potential scheme is proposed to generate complete sets of entangled photons in the context of cavity quantum electrodynamics (QED). The scheme includes twice interactions of atoms with cavities, in which the first interaction is made in two-mode optical cavities and the second one exists in a microwave cavity. In the optical cavities the atoms are resonant with the cavity modes, while the detuned interaction of the atoms with a single-mode of the microwave cavity is driven by a classical field. We show that our scheme is carried out with higher efficiency than previeous schemes, and is close to the reach of current technique.
\end{abstract}

PACS numbers: 03.67.Mn, 42.50.Dv, 03.65.Ud

*Electronic address: mangfeng1968@yahoo.com 
Entanglement is not only an essential resource for quantum information processing, such as quantum key distribution [1], quantum dense coding [2] and quantum teleportation [3], but also a key ingredient for the test of quantum nonlocality [4, 5, 6]. Many current efforts are paid on the controlled generation and detection of entangled states. Among all physical realizations of qubits, photons constitute one of the most promising systems for implementation of quantum information science, because the polarized states of the photons are individually controllable and their quantum coherence can be preserved over kilometers in an optical fiber. Most experimental realizations to generate entangled photons come almost exclusively from parametric down-conversion in nonlinear crystals [7]. Besides, one can also entangle photons by making use of atomic cascade decay [8] or excitonic emission in semiconductor quantum $\operatorname{dots}[9,10,11,12]$.

Cavity QED is an excellent technique to achieve few-qubit entanglement and quantum computing, for example, Bell state preparation [13] and two-qubit quantum gates [14]. Recently there have been some proposals to generate entangled photons in cavity QED [15, 16]. In this paper, we propose an alternative scheme for deterministically generating entangled photons in cavity QED by two steps for projecting the entanglement from atomic states to the photons emitted from these atoms. In the first step we generate photons by sending atoms through an array of optical cavities with each of two orthogonally polarized modes. In the second step the atoms are entangled by a detuned interaction with a single-mode microwave cavity. The favorable features of our scheme include: (1) It is very straightforward in implementation because we carry out the scheme by only sending atoms through the cavities. The requirement for the implementation is very close to the reach of current cavity QED techniques. (2) The complete set of the entangled two- or more-photon states can be generated deterministically by our scheme, and the implementation time remains constant with the size of the entangled photon states. (3) Our scheme is more efficient than previous proposals with cavities [15, 16], and the generated photons may be collected much more efficiently, due to cavities, than previous proposals by spontaneous emission 10, 11, 12.

We first consider the simplest case, i.e., creating the EPR photon pairs $\left|\phi^{ \pm}\right\rangle=\frac{1}{\sqrt{2}}\left(\left|\sigma^{+} \sigma^{+}\right\rangle \pm\left|\sigma^{-} \sigma^{-}\right\rangle\right)$and $\left|\psi^{ \pm}\right\rangle=$ $\frac{1}{\sqrt{2}}\left(\left|\sigma^{+} \sigma^{-}\right\rangle \pm\left|\sigma^{-} \sigma^{+}\right\rangle\right)$. As shown in Fig. 1(a), we consider two atoms going through two optical cavities (i.e., cavities 1 and 2), respectively and resonantly interacting with the cavity modes. Then they simultaneously go through a single-mode microwave cavity (i.e., the cavity 3) by detuned interaction with the cavity mode, driven by a classical field. Each of the atoms consists of two ground levels $|g\rangle$ and $|e\rangle$, and an excited level $|r\rangle$ (See Fig. 1(b)).

Let us first consider the first step of the scheme regarding the optical cavities. We assume that the cavities are initially empty and the atoms are in state $|r\rangle$, i.e., $|\psi(0)\rangle_{j}=\left|r, 0_{L}, 0_{R}\right\rangle_{j}$ with $|\cdots\rangle_{j}$ denoting the atomic state, the left and right modes of the optical cavity $\mathrm{j}$, respectively. The initial preparation of the atomic states in exited states $|r\rangle_{j}$ could be made by pumping from the corresponding ground states by some resonant $\pi$-polarization lasers before the scheme gets started. Under the rotating-wave approximation, we have following Hamiltonian in units of $\hbar=1$,

$$
\begin{aligned}
H_{j} & =\frac{1}{2}\left[\left(h_{j R} a_{j R}^{+}|g\rangle_{j j}\left\langle r\left|+h_{j L} a_{j L}^{+}\right| e\right\rangle_{j j}\langle r|\right)+h . c .\right] \\
& -i \frac{\gamma}{2}|r\rangle_{j j}\langle r|-i \kappa_{j}\left(a_{j L}^{+} a_{j L}+a_{j R}^{+} a_{j R}\right),
\end{aligned}
$$

where $h_{j R}$ and $h_{j L}$ are, respectively, the coupling strengths of the jth atom to the right-circularly $\left(\sigma^{+}\right)$and leftcircularly $\left(\sigma^{-}\right)$polarized modes of the cavity field. $a_{j L}$ and $a_{j R}\left(a_{j L}^{+}\right.$and $\left.a_{j R}^{+}\right)$are the annihilation (creation) operators of the left-circularly and right-circularly polarized modes of the jth cavity, respectively. $\gamma$ is regarding the spontaneous emission of the excited state $|r\rangle$ and $\kappa_{j}$ accounts for the decay rate of the jth cavity. For simplicity, we assume $\kappa_{j R}=\kappa_{j L}=\kappa_{j}$ and $\gamma_{1}=\gamma_{2}=\gamma$. Before any photon leaks out of each cavity, we may solve Eq. (1) following the solutions in [16, 17]. After an interaction time $\tau_{j}$, the system evolves to the entangled state,

$$
|\psi(t)\rangle_{j}=\frac{\exp \left(-\frac{\kappa_{j}+\frac{\gamma}{2}}{2} \tau_{j}\right)}{2 \Omega_{j}}\left(a\left|r, 0_{L}, 0_{R}\right\rangle_{j}+b\left|g, 0_{L}, 1_{R}\right\rangle_{j}+c\left|e, 1_{L}, 0_{R}\right\rangle_{j}\right)
$$

with

$$
\begin{aligned}
a & =\left[\left(\kappa_{j}-\frac{\gamma}{2}\right) \sin \left(\Omega_{j} \tau_{j}\right)+2 \Omega_{j} \cos \left(\Omega_{j} \tau_{j}\right)\right], \\
b & =-i h_{j R} \sin \left(\Omega_{j} \tau_{j}\right), \\
c & =-i h_{j L} \sin \left(\Omega_{j} \tau_{j}\right), \\
\Omega_{j} & =\frac{1}{2} \sqrt{2 \gamma \kappa_{j}+h_{j R}^{2}+h_{j L}^{2}-\left(\kappa_{j}+\frac{\gamma}{2}\right)^{2}} .
\end{aligned}
$$

We may carefully control the atoms' velocities to make the interaction time $\tau_{j}$ satisfy $\tan \left(\Omega_{j} \tau_{j}\right)=2 \Omega_{j} /\left(\frac{\gamma}{2}-\kappa_{j}\right)$, 
which yields Eq. (2) reducing to

$$
|\psi\rangle_{j}=\frac{1}{\sqrt{h_{j R}^{2}+h_{j L}^{2}}}\left(h_{j R}\left|g, 0_{L}, 1_{R}\right\rangle_{j}+h_{j L}\left|e, 1_{L}, 0_{R}\right\rangle_{j}\right),
$$

with the success probability

$$
P_{j}=\exp \left[-\left(\kappa_{j}+\frac{\gamma}{2}\right) \tau_{j}\right] \sin ^{2}\left(\Omega_{j} \tau_{j}\right)\left(\sqrt{h_{j R}^{2}+h_{j L}^{2}} / 2 \Omega_{j}\right)^{2} .
$$

When $t>\max \left\{1 /\left(\kappa_{1}+\frac{\gamma}{2}\right), 1 /\left(\kappa_{2}+\frac{\gamma}{2}\right)\right\}$, photons will leak out of the cavities, and the total system evolves into

$$
|\Phi\rangle=\prod_{j=1}^{2} \frac{1}{\sqrt{h_{j R}^{2}+h_{j L}^{2}}}\left(h_{j R}|g\rangle_{j}\left|\sigma^{+}\right\rangle+h_{j L}|e\rangle_{j}\left|\sigma^{-}\right\rangle\right) .
$$

To entangle the two emitted photons based on Eq. (5), we must entangle the two atoms, which is the task of the second step. Consider that the two atoms get out of the optical cavities 1 and 2, respectively, and then simultaneously get in the cavity 3 , which is a single-mode microwave cavity. We suppose that the two atoms are detuned from the mode of the cavity 3 , driven by a classical field. The Hamiltonian is 18

$$
H_{2}=\omega_{0} \sum_{j=1,2} S_{z, j}+\omega_{c} a^{+} a+G \sum_{j=1,2}\left(e^{-i \omega t} S_{j}^{+}+e^{i \omega t} S_{j}^{-}\right)+g \sum_{j=1,2}\left(S_{j}^{+} a+S_{j}^{-} a^{+}\right),
$$

where $S_{z, j}=\left(|e\rangle_{j j}\langle e|-| g\rangle_{j j}\langle g|\right) / 2, S_{j}^{+}=|e\rangle_{j j}\langle g|$, and $S_{j}^{-}=|g\rangle_{j j}\langle e|$, with $|e\rangle_{j}$ and $|g\rangle_{j}(\mathrm{j}=1$ and 2) being states in Fig. 1(b) of the jth atom. $a^{+}$and $a$ are the creation and annihilation operators for the microwave cavity mode, respectively. $\omega_{0}, \omega_{c}$ and $\omega$ are, respectively, frequencies with respect to resonant transition between levels $|e\rangle$ and $|g\rangle$, to the microwave cavity and to the classical driving field. $g$ and $G$ are the coupling constants of each atom to the cavity mode and to the driving field, respectively. We assume that $\omega_{0}=\omega$, and the rotating-wave approximation yields an effective Hamiltonian in the rotating framework with respect to the interaction-free part of Eq. (6),

$$
H_{2}^{\prime}=G \sum_{j=1,2}\left(S_{j}^{+}+S_{j}^{-}\right)+g \sum_{j=1,2}\left(e^{i \delta t} S_{j}^{+} a+e^{-i \delta t} S_{j}^{-} a^{+}\right)
$$

where $\delta=\omega_{0}-\omega_{c}$. By defining the dressed states $| \pm\rangle_{j}=\frac{1}{\sqrt{2}}\left(|g\rangle_{j} \pm|e\rangle_{j}\right)$, we make a further rotating transformation with respect to the terms regarding $G$ in Eq. (7), and obtain

$$
H_{2}^{i}=\frac{g}{2} \sum_{j=1,2}\left(|+\rangle_{j j}\langle+|-|-\rangle_{j j}\langle-|+e^{i 2 G t} \sigma_{j}^{+}-e^{-i 2 G t} \sigma_{j}^{-}\right) e^{i \delta t} a+H . c .,
$$

where $\sigma_{j}^{+}=|+\rangle_{j j}\langle-|$, and $\sigma_{j}^{-}=|-\rangle_{j j}\langle+|$. In the strong driving regime $G \gg\{\delta, g\}$, we may neglect the fast oscillating terms regarding $e^{ \pm i 2 G t}$ and get

$$
H_{2}^{I}=\frac{g}{2} \sum_{j=1,2}\left(S_{j}^{+}+S_{j}^{-}\right)\left(e^{i \delta t} a+e^{-i \delta t} a^{+}\right)
$$

In the case of $\delta \gg g / 2$ there is no energy exchange between the atomic system and the cavity. So the possible energyconserving transitions are between the states $|e\rangle_{1}|g\rangle_{2}|n\rangle$ and $|g\rangle_{1}|e\rangle_{2}|n\rangle$, and between $|e\rangle_{1}|e\rangle_{2}|n\rangle$ and $|g\rangle_{1}|g\rangle_{2}|n\rangle$, mediated by virtually excited cavity states $|n \pm 1\rangle$. Since the transition paths in both $|g\rangle_{1}|e\rangle_{2}|n\rangle \leftrightarrow|e\rangle_{1}|g\rangle_{2}|n\rangle$ and $|e\rangle_{1}|e\rangle_{2}|n\rangle \leftrightarrow|g\rangle_{1}|g\rangle_{2}|n\rangle$ interfere destructively, the effective coupling constant $\lambda$ is independent of the photon number in the cavity [19],

$$
\begin{aligned}
\lambda & =2\left(\frac{{ }_{1}\left\langle\left. g\right|_{2}\left\langle e\left|\left\langle n\left|H_{2}^{I}\right| g\right\rangle_{1}\right| g\right\rangle_{2} \mid n+1\right\rangle_{1}\left\langle\left. g\right|_{2}\left\langle g\left|\left\langle n+1\left|H_{2}^{I}\right| e\right\rangle_{1}\right| g\right\rangle_{2} \mid n\right\rangle}{\delta}\right. \\
& \left.+\frac{1\left\langle\left. g\right|_{2}\left\langle e\left|\left\langle n\left|H_{2}^{I}\right| e\right\rangle_{1}\right| e\right\rangle_{2} \mid n-1\right\rangle_{1}\left\langle\left. e\right|_{2}\left\langle e\left|\left\langle n-1\left|H_{2}^{I}\right| e\right\rangle_{1}\right| g\right\rangle_{2} \mid n\right\rangle}{-\delta}\right) \\
& =g^{2} / 2 \delta
\end{aligned}
$$


The same coupling constant $\lambda$ can be obtained from the transitions between $|e\rangle_{1}|e\rangle_{2}|n\rangle$ and $|g\rangle_{1}|g\rangle_{2}|n\rangle$, intermediated by virtually excited cavity states $|n \pm 1\rangle$. The Stark shift for the state $|e\rangle_{j}$ is

$$
\begin{aligned}
\lambda^{\prime} & =\frac{{ }_{j}\left\langle e\left|\left\langle n\left|H_{2}^{I}\right| g\right\rangle_{j}\right| n+1\right\rangle_{j}\left\langle g\left|\left\langle n+1\left|H_{2}^{I}\right| e\right\rangle_{j}\right| n\right\rangle}{\delta} \\
& +\frac{{ }_{j}\left\langle e\left|\left\langle n\left|H_{2}^{I}\right| g\right\rangle_{j}\right| n-1\right\rangle_{j}\left\langle g\left|\left\langle n-1\left|H_{2}^{I}\right| e\right\rangle_{j}\right| n\right\rangle}{-\delta} \\
& =g^{2} / 4 \delta,
\end{aligned}
$$

where $\mathrm{j}=1$ and 2 . There same values for the case of $|g\rangle_{j}$ and then $H_{2}^{I}$ turns to

$$
H_{2}^{e f f}=\lambda^{\prime} \sum_{j=1,2}\left(|e\rangle_{j j}\langle e|+| g\rangle_{j j}\langle g|\right)+\lambda\left(S_{1}^{+} S_{2}^{+}+S_{1}^{+} S_{2}^{-}+H . c .\right),
$$

where the effective coupling constant $\lambda$ and $\lambda^{\prime}$ are both independent of the photon number of the cavity field. If the two atoms are in the state in Eq. (5) before getting in the microwave cavity, a straightforward algebra leads to the evolved state of the total system [20],

$$
\begin{aligned}
& \widetilde{N}\left[|g g\rangle\left(h_{1 R} h_{2 R}\left|\sigma^{+} \sigma^{+}\right\rangle-i h_{1 L} h_{2 L}\left|\sigma^{-} \sigma^{-}\right\rangle\right)\right. \\
& -i|e e\rangle\left(h_{1 R} h_{2 R}\left|\sigma^{+} \sigma^{+}\right\rangle+i h_{1 L} h_{2 L}\left|\sigma^{-} \sigma^{-}\right\rangle\right) \\
& +|g e\rangle\left(h_{1 R} h_{2 L}\left|\sigma^{+} \sigma^{-}\right\rangle-i h_{1 L} h_{2 R}\left|\sigma^{-} \sigma^{+}\right\rangle\right) \\
& \left.-i|e g\rangle\left(h_{1 R} h_{2 L}\left|\sigma^{+} \sigma^{-}\right\rangle+i h_{1 L} h_{2 R}\left|\sigma^{-} \sigma^{+}\right\rangle\right)\right],
\end{aligned}
$$

where $\widetilde{N}=\frac{1}{\sqrt{2} \sqrt{h_{1 R}^{2}+h_{1 L}^{2}} \sqrt{h_{2 R}^{2}+h_{2 L}^{2}}}$ is the normalization constant, and we have chosen $\lambda t=\pi / 4$, and $G t=n \pi$, with $\mathrm{n}$ being an integer. For simplicity, we only consider the case of $h_{j R}=h_{j L}=h_{j}$. So we have

$$
\begin{aligned}
& (1 / 2 \sqrt{2})\left[|g g\rangle\left(\left|\sigma^{+} \sigma^{+}\right\rangle-i\left|\sigma^{-} \sigma^{-}\right\rangle\right)-i|e e\rangle\left(\left|\sigma^{+} \sigma^{+}\right\rangle+i\left|\sigma^{-} \sigma^{-}\right\rangle\right)\right. \\
& \left.-i|e g\rangle\left(\left|\sigma^{+} \sigma^{-}\right\rangle+i\left|\sigma^{-} \sigma^{+}\right\rangle\right)+|g e\rangle\left(\left|\sigma^{+} \sigma^{-}\right\rangle-i\left|\sigma^{-} \sigma^{+}\right\rangle\right)\right] .
\end{aligned}
$$

Eq. (11) presents a deterministic generation of entangled photon pairs with our scheme, while to obtain a certain entangled photon pairs, we only have the success rate $1 / 4$. Moreover, the classical driving field can be removed from the second step in above implementation, which actually corresponds to the experimental implementation in [13]. But in that case, only $\left|\psi^{ \pm}\right\rangle$, instead of a complete set of Bell states in Eq. (11), could be obtained.

Our scheme is suitable for not only repeatedly producing entangled photon pairs, but also generating entangled multiphotons. For the latter case, after, for example, $\mathrm{N}$ atoms are sent through $\mathrm{N}$ two-mode optical cavities, i.e., cavities 1, 2, $\cdots, \mathrm{N}$ in Fig. 1(c), respectively, a careful control of the interaction time $\tau_{j}$ to satisfy $\tan \left(\Omega_{j} \tau_{j}\right)=$ $2 \Omega_{j} /\left(\frac{\gamma}{2}-\kappa_{j}\right)$ would lead to a state,

$$
|\Phi\rangle=\prod_{j=1}^{N} \frac{1}{\sqrt{2}}\left(|g\rangle_{j}\left|\sigma^{+}\right\rangle+|e\rangle_{j}\left|\sigma^{-}\right\rangle\right),
$$

where we have assumed $h_{j R}=h_{j L}=h_{j}(\mathrm{j}=1,2, \cdots, \mathrm{N})$ for simplicity. A convenient treatment for an ensemble of spin-1/2 atoms is to use the collective spin operators. By transforming the atomic states to eigenstates of a collective operator $S_{x}$ with

$$
S_{x}=\frac{1}{2} \sum_{j=1}^{N}\left(S_{j}^{+}+S_{j}^{-}\right),
$$

we may get the following effective Hamiltonian in the interaction picture related to Eq. (6),

$$
H=\left(i \frac{\partial}{\partial t} U\right) U^{+}+U H_{N}^{e f f} U^{+}=2 G S_{x}+2 \lambda S_{x}^{2},
$$

where $U=e^{-i 2 G S_{x} t}$, and $H_{N}^{e f f}$ is of similar form to $H_{2}^{e f f}$ but for $\mathrm{N}$ atoms. We have noticed a recent work [19] for entangling many trapped ions by using angular momentum representation with rotating basis states $|N / 2, M\rangle_{x}, \mathrm{M}$ 
$=-\mathrm{N} / 2, \ldots, \mathrm{N} / 2$, with respect to the atomic states. Since $|N / 2, M\rangle_{x}$ are the eigenstates of $S_{x}$, by using Eq. (13), we could obtain the analytical form of the entangled ionic states by direct algebra. In our case, however, due to the degenerate states, the basis states above are not sufficient for a complete subspace. So we have to introduce an additional degree of freedom $\mathrm{K}$ to lift the degeneracy, and thereby the basis states are $|N / 2, M, K\rangle_{x}$ with $\mathrm{M}=-\mathrm{N} / 2$, $\ldots ., \mathrm{N} / 2$ and $\mathrm{K}=0,1, \ldots . \mathrm{N}$. The newly introduced $\mathrm{K}$ accounts for the number of the minus signs ' ${ }^{\prime}{ }^{\prime}$ contributed from the excited atomic levels. So for a state with $n_{e}$ components regarding the excited atomic level, we have

$$
|g \ldots g \underbrace{e \ldots e}_{n_{e}}\rangle=\prod_{M, K} C_{M K}(-1)^{N / 2-M}|N / 2, M, K\rangle_{x},
$$

where $\mathrm{M}=-\mathrm{N} / 2, \ldots . \mathrm{N} / 2, \mathrm{~K}=0,1, \ldots, n_{e}, C_{M K}=(-1)^{K} C_{M}$ and $C_{M}$ is given in [19]. A complete discussion about the properties of this full set of basis states could be found in 21]. In the present paper, however, our interest is only in the generation of entangled states based on these states. Considering current experimental feasibility and also for clarity, we will below demonstrate a generation of three entangled photons in detail by a formally simpler solution than in 21]. For the Hamiltonian Eq. (13) with $\mathrm{N}=3$, we transform the atomic states into the eigenstates of $S_{x}$, and obtain,

$$
\left(\begin{array}{l}
\mid \text { ggg }\rangle \\
\mid \text { gge }\rangle \\
\mid \text { geg }\rangle \\
\mid \text { gee }\rangle \\
\mid \text { egg }\rangle \\
\mid \text { ege }\rangle \\
|e e g\rangle \\
\mid \text { eee }\rangle
\end{array}\right)=\frac{1}{8}\left(\begin{array}{cccccccc}
1 & 1 & 1 & 1 & 1 & 1 & 1 & 1 \\
1 & -1 & 1 & -1 & 1 & -1 & 1 & -1 \\
1 & 1 & -1 & -1 & 1 & 1 & -1 & -1 \\
1 & -1 & -1 & 1 & 1 & -1 & -1 & 1 \\
1 & 1 & 1 & 1 & -1 & -1 & -1 & -1 \\
1 & -1 & 1 & -1 & -1 & 1 & -1 & 1 \\
1 & 1 & -1 & -1 & -1 & -1 & 1 & 1 \\
1 & -1 & -1 & 1 & -1 & 1 & 1 & -1
\end{array}\right)\left(\begin{array}{l}
|+++\rangle \\
|++-\rangle \\
|+-+\rangle \\
|+--\rangle \\
|-++\rangle \\
|-+-\rangle \\
|--+\rangle \\
|---\rangle
\end{array}\right)
$$

By choosing $G t=(2 n+3 / 4) \pi$ and $\lambda t=\pi / 4$, we have following evolution of the three-atom states.

$$
\begin{aligned}
|g g g\rangle & \Longrightarrow \frac{1}{\sqrt{2}}(|g g g\rangle+i|e e e\rangle), \quad|g g e\rangle \Longrightarrow \frac{1}{\sqrt{2}}(|g g e\rangle+i|e e g\rangle), \\
|g e g\rangle & \Longrightarrow \frac{1}{\sqrt{2}}(|g e g\rangle+i|e g e\rangle), \quad|g e e\rangle \Longrightarrow \frac{1}{\sqrt{2}}(|g e e\rangle+i|e g g\rangle), \\
|e g g\rangle & \left.\Longrightarrow \frac{1}{\sqrt{2}}(|e g g\rangle+i|g e e\rangle), \quad|e g e\rangle \Longrightarrow \frac{1}{\sqrt{2}}(\mid \text { ege }\rangle+i|g e g\rangle\right), \\
|e e g\rangle & \left.\Longrightarrow \frac{1}{\sqrt{2}}(|e e g\rangle+i|g g e\rangle), \quad|e e e\rangle \Longrightarrow \frac{1}{\sqrt{2}}(\mid \text { eee }\rangle+i|g g g\rangle\right),
\end{aligned}
$$

where we have discarded their common global phase. Therefore, if the atoms are in a state in Eq. (12) with $\mathrm{N}=3$, after they go through the microwave cavity simultaneously, a certain detection on the atomic internal states would yield a certain state from the complete set of three-photon entangled states.

Following above algebra, we may have a similar state evolution to Eq. (16), after lengthy but straightforward deduction, for $\mathrm{N}$ atoms prepared in Eq. (12) and sent through the microwave cavity simultaneously, under the conditions $G t=(2 n+3 / 4) \pi$ and $\lambda t=\pi / 4$ when $\mathrm{N}$ is odd, or under the conditions $G t=n \pi$ and $\lambda t=\pi / 4$ when $\mathrm{N}$ is even.

To get a highly efficient generation of entangled photons, we require $\frac{\gamma}{2}<\kappa_{j}<h_{j}$. In optical cavities, the singlephoton coupling strength $h=2 \pi \times 34 \mathrm{MHz}$, the atomic decay rate $\gamma=2 \pi \times 2.6 \mathrm{MHz}$ and the cavity decay rate $\kappa=2 \pi \times 4.1 \mathrm{MHz}$ have been reported [22, 23]. So in the case of two atoms, by setting $\kappa_{1}=\kappa_{2}=\kappa$, and $h_{1}=h_{2}=h$, we get the success probability to be $P=P_{1} \times P_{2}=48.1 \%$ at $\tau_{j}=10.8 \mathrm{~ns}$. The efficiency can be higher with smaller parameters $\gamma$ and $\kappa$, as shown in Fig. 2. In the second step, we assume the coupling of the atoms with the microwave cavity to be $g \simeq 2 \pi \times 50 \mathrm{kHz}$, and the photon storage time in a microwave cavity to be $T_{c}=1 \mathrm{~ms}$ (corresponding to $\left.\mathrm{Q}=3 \times 10^{8}\right)$ [24]. So with the choice $\delta=5 \mathrm{~g}$, the interaction time of the atom with the cavity field is of the order of tenth of microsec.

Due to the large detuning employed in the second step, the success rate of the photon generation under the detrimental influence of the cavity decay in our scheme is only related to the optical cavities. In this sense, we may make a comparison of our operations in the optical cavity with those in [15. Because of the near resonance and also because the generation of the second photon is based on that of the first photon, a relatively big cavity decay rate is required in [15]. In contrast, our scheme goes better with smaller cavity decay. So it is understandable that our scheme is of much higher success rate than that (i.e., approximate 0.06) in 15] (See Fig. 2). More importantly, our 
scheme can be directly extended to the preparation of many-photon entanglement, while this is impossible in [15. The recent proposal based on a magnetic field gradient also enables a generation of multiphoton entangled states by mapping entanglement from atoms to photons. But it is much slower than our present scheme. It generates an entangled photon pair by a time of the order of milisec, and the implementation time would be much longer for producing entangled states of more photons. In contrast, no matter how many photons would be entangled by our scheme, the implementation time remains constant.

To the best of our knowledge two atoms interacting coherently in a microwave cavity have been achieved experimentally [13], while we have not yet found any experimental report for more than two atoms controllably interacting in a microwave cavity. A big challenge for an experimental realization of our scheme is to sent the atoms through the microwave cavity simultaneously, and any deviation from the simultaneousness would lead to infidelity. We have assessed the infidelity due to operational imperfection in Fig. 3 for the cases of two and three atoms. For simplicity, we suppose that the atoms are moving with the same speed, but enter the microwave cavity sequentially with the time difference $\delta t$ between neighboring atoms. For the two-atom case, the interaction time of the two atoms with the detuned cavity mode is thereby reduced to $\left(t_{0}-2 \delta t\right)$ with $t_{0}$ the desired time in the ideal case. In addition to the single atom resonantly interacting with the classical driving field during the time interval $\delta t$, as well as free evolution of the atoms, we can obtain the infidelity, due to the operational imperfection, to be $\sin ^{2}(G \delta t)+\cos ^{2}(G \delta t)\{1-$ $\sin [2 \lambda(t-2 \delta t)]\} / 2$. Similar consideration on the three-atom case yields a more complicated analytical result for the infidelity which omitted here. The numerical calculation in Fig. 3 could tell us that the infidelity would be increasing with the atom number and $\delta t$, while our scheme works well if $\delta t<0.01 t_{0}$ in cases of $\mathrm{N}=2$ and 3 . On the other hand, in the case of the atoms with different speeds, the infidelity is also obtainable, similar to the result to the above discussion for non-simultaneous movement of the atoms, if the two atoms get in the microwave cavity at the same time. As one atom will go out of the microwave cavity before the other one, the time deviation from the desired time will yield a single atom resonantly interacting with the classical driving field. But if the atoms going through the respective optical cavities with different times from the desired ones, i.e., not meeting the condition $\tan \left(\Omega_{j} \tau_{j}\right)=2 \Omega_{j} /\left(\frac{\gamma}{2}-\kappa_{j}\right)$, then additional infidelity will be yielded. So, in principle, the situation for atoms with different speeds is worse than that for atoms with the same speed but without simultaneous movement in the microcavity.

It is evident that our scheme is still challenging experimentally. First of all, our requirement for optical cavities with two orthogonal modes of different frequencies has not yet achieved experimentally so far. But we have noticed significant advances in recent experiments [22, 23] with optical cavities including strong coupling of atoms and identification of individual atoms, which implies that a single atom going through an optical cavity is controllable. Secondly, for atoms sent through a microwave cavity simultaneously, we have noticed that, even in the two-atom case, the achieved experiment [13] was done by using van der Waals collision of the atoms in the central area of the microwave cavity, instead of sending the atoms through the microwave cavity strictly simultaneously. While this experimental discrepancy from the theoretical design did not hamper further proposals for quantum information processing with cavity QED based on simultaneously sending many atoms through a cavity [14, 25]. Like those proposals, we propose this scheme also based on the expectation that the above mentioned difficulties in experiments would be overcome in the future with more advances in cavity QED techniques.

As a final remark, we emphasize that the photons entangled in polarization but with different energies should be as useful as those entangled identical photons in quantum information processing, if our implementation is only on the polarized degrees of freedom. A previous scheme based on biexcitons in semiconductor quantum dots also produces entanglement of two photons with different energies [9].

In conclusion, we have proposed a potential scheme for creating complete sets of entangled two- or more-photon states in the context of cavity QED, which is more efficient than recent proposals [15, 16]. In contrast to previous proposals for generating entangled photons by spontaneous emission, our scheme carried out by means of cavities would be of much higher rate for photon collection. This is of significant importance in view of the application of entangled photon source. Moreover, our scheme enables a deterministic generation of entangled multiphoton states, which is also more efficient than by stochastic method with parametric down-conversion. More importantly, our scheme is close to the reach of current techniques of cavity QED.

This work is supported by National Natural Science Foundation of China under Grants No. 10474118 and No. 10274093, by Hubei provincial funding for distinguished youth, and by the National Fundamental Research Program of China under Grant No. 2005CB724502.

[1] Ekert A K 1991 Phys. Rev. Lett. 67661.

[2] Bennett C H and Wiesner S J 1992 Phys. Rev. Lett. 69, 2881.

[3] Bennett C H et al. 1993 Phys. Rev. Lett. 70, 1895.

[4] Einstein A et al. 1935 Phys. Rev. 47, 777. 
[5] Bell J S 1965 Physics (long Island City, N.Y.) 1, 195.

[6] Greenberger D M et al 1990 Am. J. Phys. 58, 1131.

[7] Kwiat P G et al. 1995 Phys. Rev. Lett. 75, 4337.

[8] Aspect A et al. 1982 Phys. Rev. Lett. 49, 91.

[9] Benson O et al. 2000 Phys. Rev. Lett. 84, 2513.

[10] Cerletti V et al. eprint quant-ph/0411235.

[11] Titov $M$ et al. eprint cond-mat/0503676

[12] Edamatsu K et al. 2004 Nature 431, 167.

[13] Osnaghi S et al. 2001 Phys. Rev. Lett. 87, 037902.

[14] Yamaguchi F et al. 2002 Phys. Rev. A 66, 010302(R).

[15] Zhou D L et al. 2005 Phys. Rev. A 72, 040302(R).

[16] Feng M et al. 2005 Phys. Rev. A 72, 042333.

[17] Zou X B and Mathis W 2005 Phys. Rev. A 71, 042334.

[18] Solano E et al. 2003 Phys. Rev. Lett. 90, 027903.

[19] Mølmer K and Sørensen A 1999 Phys. Rev. Lett. 82, 1835.

[20] Eq. (10) and the following Eq. (16) are actually written in the interacting picture related to Eq. (7). If returning to the Schrödinger picture, we have different phase factors between $|e\rangle$ and $|g\rangle$, which have no effect on the main result due to our detection on the atomic levels.

[21] Arecchi F T et al. 1972 Phys. Rev. A 6, 2211.

[22] McKeever J et al. 2004 Phys. Rev. Lett. 93, 143601.

[23] Boca A et al. 2004 Phys. Rev. Lett. 93, 233603.

[24] Raimond J M et al. 2001 Rev. Mod. Phys. 73, 565.

[25] Deng Z J et al. 2006 Phys. Rev. A 73, 014302 and the references therein. Captions of the figures

Fig. 1. (a) The schematics for generation of an entangled photon pair by cavity QED, where the wavy arrows denote the dissipation from the corresponding cavities. (b) The level configuration of the atoms, where $|g\rangle$ and $|e\rangle$ are hyperfine levels of the ground state with level splitting of the order of $\mathrm{GHz}$, and $|r\rangle$ is the excited level higher than the ground states by an optical frequency. $h_{j R}$ and $h_{j L}$ are coupling constants of the atom to $\sigma^{+}$and $\sigma^{-}$modes of the optical cavity. (c) The illustration of the extended scheme for generating entangled multiphoton states by cavity QED.

Fig. 2. The success probability of generation of an entangled photon pair, where the solid curve is plot with the achieved experimental parameters $h=2 \pi \times 34 \mathrm{MHz}, \gamma=2 \pi \times 2.6 \mathrm{MHz}$ and $\kappa=2 \pi \times 4.1 \mathrm{MHz}$; and the dot-dashed curve corresponds to $h=2 \pi \times 34 \mathrm{MHz}, \gamma=2 \pi \times 0.1 \mathrm{MHz}$ and $\kappa=2 \pi \times 0.6 \mathrm{MHz}$ [17]. The dashed lines denote the time points for producing the state in Eq. (3).

Fig. 3. Infidelity versus time different $\delta t$, where the dashed and solid curves represent the cases of two and three atoms, respectively, and $t_{0}$ is the desired time in the ideal situation. We assume the atoms with the exactly same speed enter the microwave cavity, but non-simultaneously with the time difference $\delta t$. 
(a)

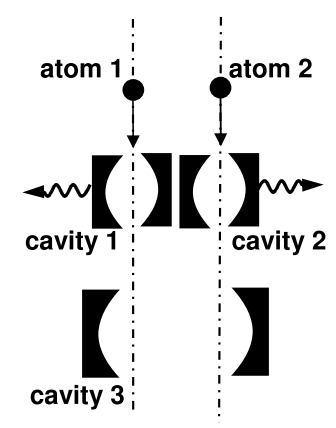

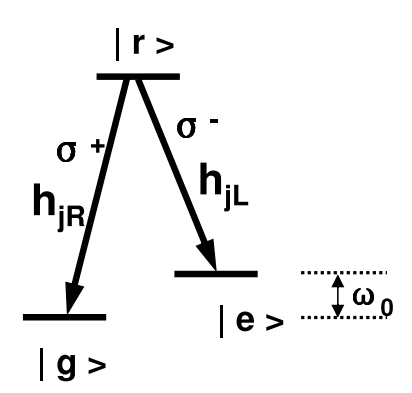

(b)

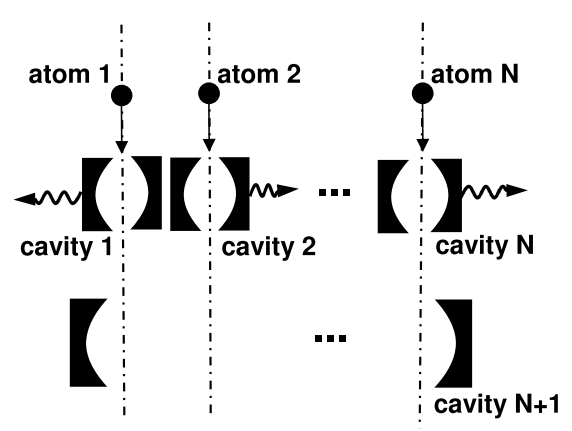

(c)

FIG. 1: Fig1 


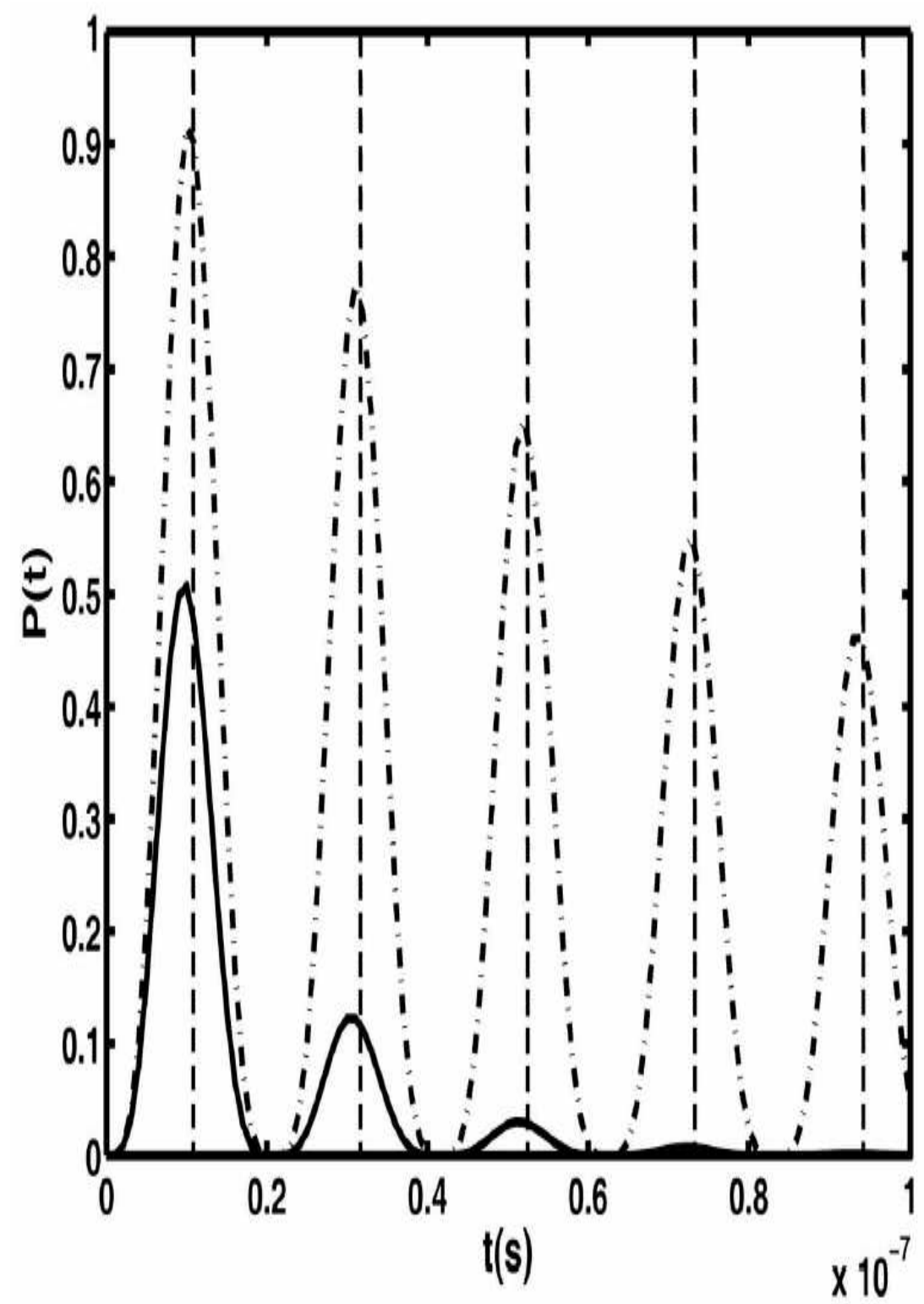

FIG. 2: Fig2 


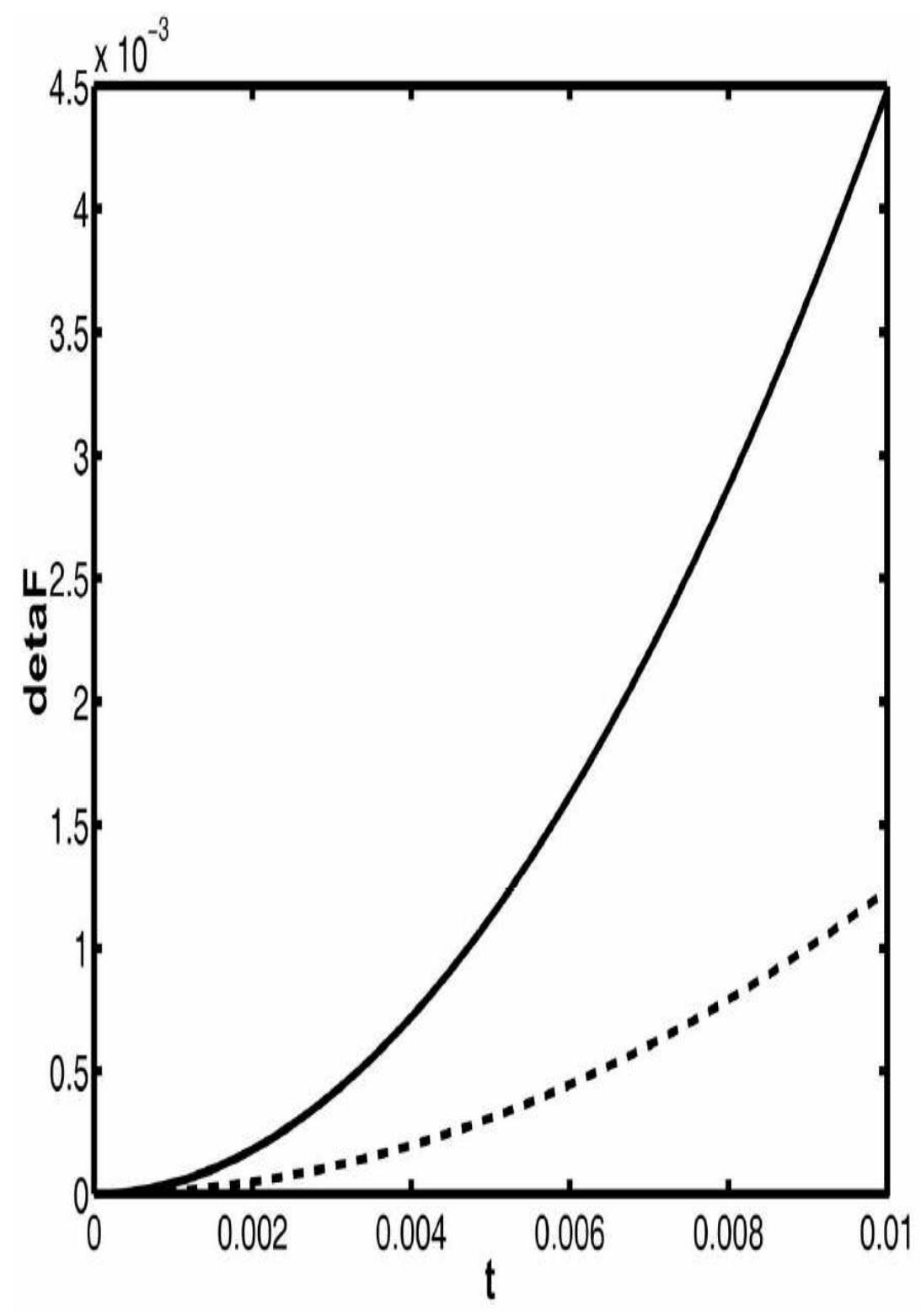

FIG. 3: Fig3 